\title{
The WURM project - a freely available web-based repository of computed physical data for minerals
}

\author{
Razvan Caracas ${ }^{1}$, Ema Bobocioiu ${ }^{2}$ \\ ${ }^{1}$ CNRS, ENS de Lyon, Univ. Claude Bernard Lyon, Laboratoire de Geologie, UMR 5276, Lyon, France \\ ${ }^{2}$ ENS de Lyon, Univ. Claude Bernard Lyon, Laboratoire de Geologie, UMR 5276, Lyon, France
}

The WURM project is a database of computed Raman and infrared spectra and other physical properties for minerals. The calculations are performed within the framework of the density-functional theory and the density-functional perturbation theory in the ABINIT implementation. The database is freely available for teaching and research purposes and is presented in a web-based format, hosted on the http://www.wurm.info website. It provides the crystal structure, the parameters of the calculations, the dielectric properties, the Raman spectra with both peak positions and intensities and the infrared spectra with peak positions for minerals. It shows the atomic displacement patterns for all the zonecenter vibrational modes and the associated Raman tensors. The web presentation is user friendly and highly oriented towards the end user, with a strong educational component in mind. A set of visualization tools ensures the observation of the crystal structure, the vibrational pattern, and the different spectra. Further developments include elastic and optical properties of minerals.

References:

Caracas, R., Bobocioiu, E. (2011), American Mineralogist, vol. 96, pp. 437-443. 